\title{
Prognostic relevance of proliferation-related miRNAs in pancreatic neuroendocrine neoplasms
}

\author{
Vince Kornél Grolmusz 1,2,3, Annamária Kövesdi 1,2, Katalin Borka4, Peter Igaz 1,5 and Attila Patócs 2,5,6 \\ ²nd Department of Medicine, Semmelweis University, Budapest, Hungary, 2`Lendület’ Hereditary Endocrine \\ Tumours Research Group, Hungarian Academy of Sciences - Semmelweis University, Budapest, Hungary, \\ ${ }^{3}$ Department of Medical Oncology and Therapeutics, Beckman Research Institute, City of Hope National Medical \\ Center, Monrovia, California, USA, ${ }^{4}$ 2nd Department of Pathology, Semmelweis University, Budapest, Hungary, \\ ${ }^{5}$ MTA-SE Molecular Medicine Research Group, Hungarian Academy of Sciences - Semmelweis University, Budapest, \\ Hungary, and ${ }^{6}$ Department of Laboratory Medicine, Semmelweis University, Budapest, Hungary \\ Correspondence \\ should be addressed \\ to A Patócs \\ Email \\ patocs.attila@med. \\ semmelweis-univ.hu
}

\begin{abstract}
Objective: Pancreatic neuroendocrine neoplasms (PanNENs) are rare tumors arising from the endocrine pancreas; however, their prognosis differs significantly upon their proliferative state, which is characterized by histopathological grading. MiRNAs are small, noncoding RNAs posttranscriptionally regulating gene expression. Our aim was to identify miRNAs with altered expression upon proliferation which can be used as prognostic biomarkers in PanNENs. Methods: MiRNA expression profiles of 40 PanNENs were downloaded from Gene Expression Omnibus and were reanalyzed upon tumor grades (discovery cohort). Results of the reanalysis were confirmed by qRT-PCR analysis of five miRNAs on an independent validation cohort of 63 primary PanNEN samples. Cox proportional hazards survival regression models were fit for both univariate and multivariate analysis to determine the miRNAs' effect on progression-free and overall survival.

Results: Nineteen miRNAs displayed differential expression between tumor grades. The altered expression of three out of five chosen miRNAs was successfully validated; hsa-miR-21, hsa-miR-10a and hsa-miR-106b were upregulated in more proliferative PanNENs compared to Grade 1 tumors. In univariate analysis, higher expression of tissue hsamiR-21, hsa-miR-10a and hsa-miR-106b of primary PanNENs predicted worse progression-free and overall survival; however, multivariate analysis only confirmed the expression of hsa-miR-21 as an independent prognostic factor. Conclusions: The expression of hsa-miR-106b, hsa-miR-10a and especially hsa-miR-21 has prognostic relevance regarding progression-free and overall survival in patients with PanNENs.
\end{abstract}

\section{Introduction}

Pancreatic neuroendocrine neoplasms (PanNENs) are rare tumors arising from the endocrine islets of the pancreas with an incidence of 0.2-0.3/100 $000(1,2)$. In the majority of the cases, PanNENs occur sporadically; however, some hereditary tumor syndromes (e.g. multiple endocrine neoplasia type 1, von Hippel-Lindau syndrome, neurofibromatosis type 1 , tuberous sclerosis) might also result in PanNEN formation (3). Histological classification (c) 2018 European Society of Endocrinology Printed in Great Britain of these tumors is based on their proliferative nature assessed by the Ki-67 index and mitotic count (4). Upon the WHO classification, Grade 1 and Grade 2 tumors are less proliferative, while the prognosis of Grade 3 tumors is very poor (4).

High throughput analyses contributed to enlighten the molecular background of PanNENs $(5,6)$. A whole exome sequencing analysis demonstrated frequent 
mutations in genes implicated in chromatin remodeling (MEN1, DAXX, ATRX), and mutations in other well-known tumor suppressor genes (PTEN, TSC2) were also detected (5). Additionally, a gene expression microarray study performed on a large sample set confirmed the decreased expression of mTOR pathway-inhibiting PTEN and TSC2 contributing to PanNEN formation (6). Most recently, a large scale whole-genome sequencing study found that germline mutations of DNA repair genes MUTYH, CHEK2 and BRCA2 might also be found in clinically sporadic cases (7).

miRNAs are small, 21-26nt long single-stranded RNA molecules regulating gene expression at the posttranscriptional level via RNA interference (8). Several layers of evidence confirmed the role of tissue-specific miRNA expression alterations in the pathogenesis of various tumors (9). As miRNAs are chemically more stable compared to mRNAs, miRNA expression can optimally be measured from formalin-fixed, paraffin-embedded samples $(10,11,12)$, as it correlates well with expression levels obtained from fresh frozen specimens (11).

To date, only two studies examined the PanNEN miRNA expression profiles $(13,14)$. These single cohort studies proposed the well-known onco-miR hsa-miR-21 to have elevated expression in highly proliferative, metastatic PanNENs (13), while the expression of hsamiR-642 was found to be correlated with Ki-67 score and hsa-miR-210 correlated with metastatic disease (14).

Although the reanalysis of previous miRNA microarray experiments revealed three clusters of PanNENs upon miRNA expression signature (15), the prognostic significance of these clusters or single miRNAs has not been thoroughly investigated. Only one study proposed prognostic relevance of hsa-miR-196a to be associated with poorer prognosis in a sample of 37 PanNENs (16).

Here, we present a reanalysis of previous miRNA profiling data and validate our findings on an independent, larger dataset containing 63 primary tumors revealing miRNAs of prognostic importance in PanNENs.

\section{Subjects and methods}

\section{In silico reanalysis of publicly available miRNA microarray data}

Previously published miRNA expression profiles were downloaded from Gene Expression Omnibus (GEO ID: GSE73350) (13) and were reanalyzed using GeneSpring 12.6 (Agilent Technologies) software. In total, 40 miRNA expression arrays of PanNEN tissues were included (discovery cohort).

\section{PanNEN patients and tissues}

Sixty three independent, primary PanNEN samples were enrolled between 1999 and 2015 at the 2nd Department of Pathology, Semmelweis University (validation dataset). Consent has been obtained from each patient or subject after full explanation of the purpose and nature of all procedures used. All patients were of Caucasian origin. Routine pathological examination followed by histopathological analyses, including Ki-67 immunohistochemistry analysis and determination of the mitotic count performed by a pathologist expert in endocrine pathology ( $\mathrm{K} \mathrm{B}$ ) confirmed the diagnosis of PanNEN. Ki-67 immunohistochemistry was performed as earlier reported using the monoclonal mouse anti-Ki-67 antibody (Clone MIB-1, Dako Cat. No.: M7240) (10). Grading of PanNEN samples was performed according to the WHO 2017 classification with the grade denoting the higher value of Ki-67 index and mitotic count (17). The study was approved by the Scientific and Research Committee of the Medical Research Council of Hungary (ETT-TUKEB 4457/2012/EKU).

\section{RNA isolation and quantitative real-time PCR}

Total RNA was isolated from formalin-fixed, paraffinembedded sections using Ambion RecoverAll Total Nucleic Acid Isolation kit for FFPE tissues (Thermo Fisher Scientific) as earlier reported (10). Five nanograms of total RNA was reverse transcribed using single tube TaqMan microRNA assays (assay IDs: hsa-miR-21: 000397; hsamiR-29a: 002112; hsa-miR-10a: 000387; hsa-miR-106b: 000442; hsa-miR-101: 002253; RNU6B: 001093) and TaqMan microRNA reverse transcription kit (Thermo Fisher Scientific) while miRNA expression was quantified on a 7500 Fast Real-time PCR instrument (Thermo Fisher Scientific) as earlier reported (10).

MiRNA expression was normalized to the relative expression of RNU6B ( $\Delta$ Ct method) (10). All measurements were performed in triplicate.

\section{Statistical analysis}

Statistical analysis of the microarray data was performed using GeneSpring 12.6 (Agilent Technologies) software as described earlier (18). Following the manufacturer's recommendation, total signal normalization at the 75 th 
percentile of raw signal values and baseline transformation at the median of all samples were performed as described earlier (18). Differentially expressed miRNAs upon tumor grade (Grade 1, Grade 2, Grade 3) were detected by oneway ANOVA followed by Tukey's Honestly Significant Difference post hoc test and Benjamini-Hochberg correction for multiple measurements (18). Hierarchical clustering was performed on the Morpheus online tool (URL: https://software.broadinstitute.org/morpheus/) using the one minus Pearson correlation method.

The results of qRT-PCR experiments were analyzed using GraphPad Prism version 6 software. The normality of distribution was confirmed by Shapiro-Wilk normality test. For examining miRNA expression differences between tumor grades ( $-\Delta \mathrm{Ct}$ values), one-way ANOVA test and subsequent post hoc Tukey's multiple comparisons test was used. For examining miRNA expression differences between patients with non-metastatic and metastatic disease ( $-\Delta \mathrm{Ct}$ values), Student's independent samples $T$-test was used. For significantly differently expressed miRNAs both in the discovery and validation cohorts, Cox proportional hazards survival regression models were fit for both univariate and multivariate analysis to determine their effects on progression-free and overall survival and also on the time to disease progression.

In all comparisons, $P$ value $<0.05$ was considered statistically significant.

\section{Results}

\section{In silico reanalysis and subsequent validation identifies several miRNAs to be differently expressed upon tumor grade in PanNENs}

Upon the in silico reanalysis in the discovery cohort, 19 miRNAs, including the well-known onco-miR hsamiR-21, were found to have altered expression between tumor grade in PanNENs (Fig. 1, Panel A). Hierarchical clustering upon the expression profiles of differentially expressed miRNAs rendered the samples in two clusters differing in proliferative nature: $\mathrm{C} 1$ cluster-incorporated eighteen Grade 1 and four Grade 2 tumors, while C2 consisted of five Grade 1, eleven Grade 2 and two Grade 3 tumors (Fig. 1, Panel A).

Five miRNAs (hsa-miR-21, hsa-miR-29a, hsa-miR-10a, hsa-miR-106b and hsa-miR-101) were chosen for validation in the independent validation cohort containing 28 Grade 1, 27 Grade 2 and 8 Grade 3 tumors (Table 1). The validation cohort contained 44 non-functioning tumors,
17 insulinomas, one gastrinoma and one somatostatinoma and 38 out of 63 of the patients were female. The mean age of the patients was $54.4 \pm 17.3$ years. Among the five chosen miRNAs, hsa-miR-21, hsa-miR-10a and hsa-miR106b were differently expressed between tumor grades and all three were upregulated in more proliferative PanNENs (Fig. 1, Panel B).
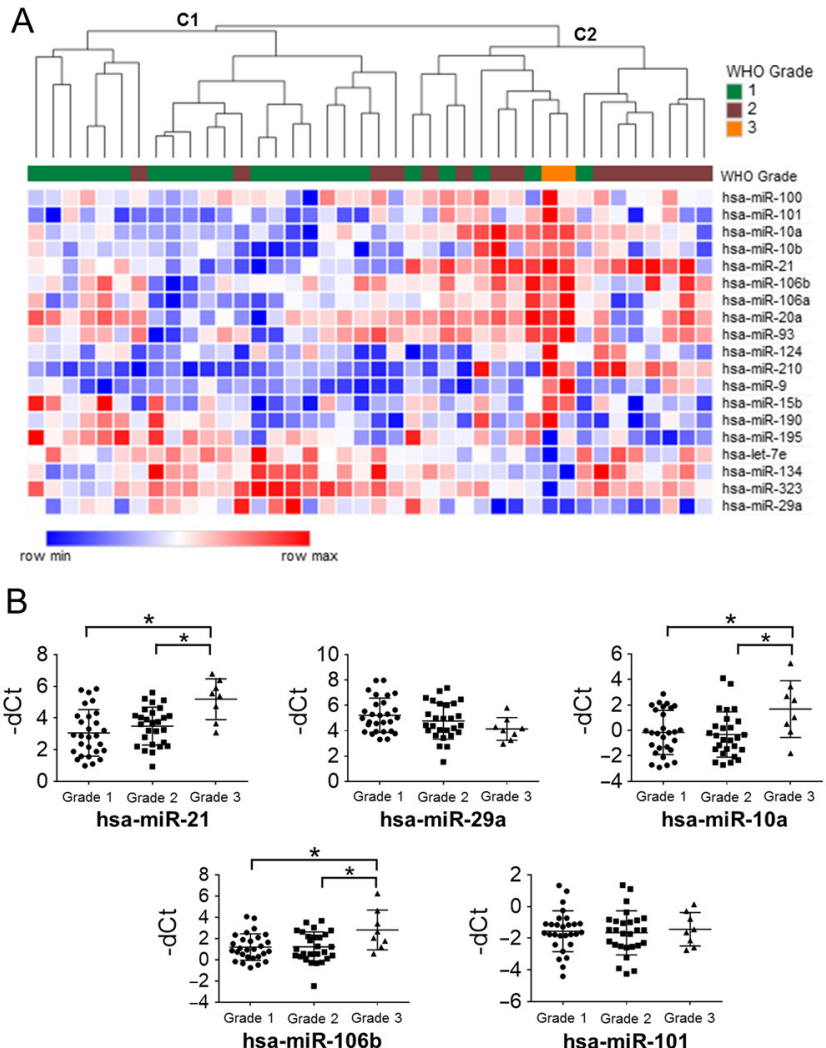

\section{Figure 1}

Discovery and validation of novel miRNAs differently expressed upon tumor grade in PanNENs. Panel (A): Heat map and hierarchical clustering of significantly differently expressed miRNAs upon tumor grade in the in silico reanalysis of the previously published dataset by Roldo et al. (13). Panel (B): Validation of the expression of five chosen miRNAs (hsa-miR-21, hsa-miR-29a, hsa-miR-10a, hsa-miR-106 and hsa-miR-101) in the validation cohort by qRT-PCR measurements. Higher $-\Delta C$ t levels correspond to higher miRNA expression. Bars indicate mean \pm standard deviation (s.D.). Statistics: one-way ANOVA followed by Tukey's multiple comparisons test. Asterisks mark statistical significance $(P<0.05)$. A full colour version of this figure is available at https://doi.org/10.1530/EJE-18-0305. 


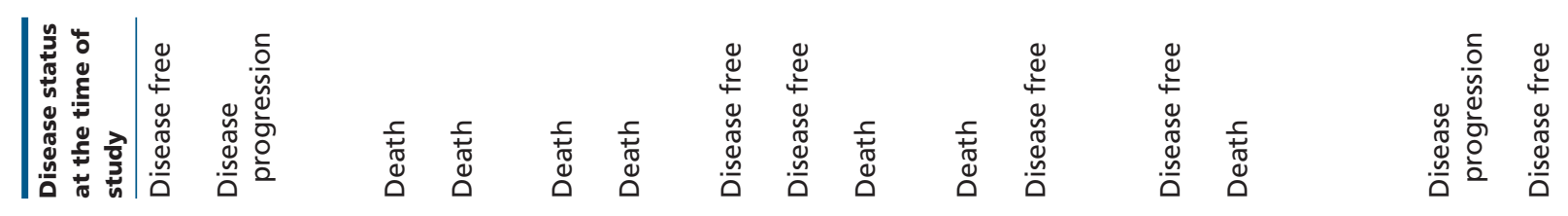

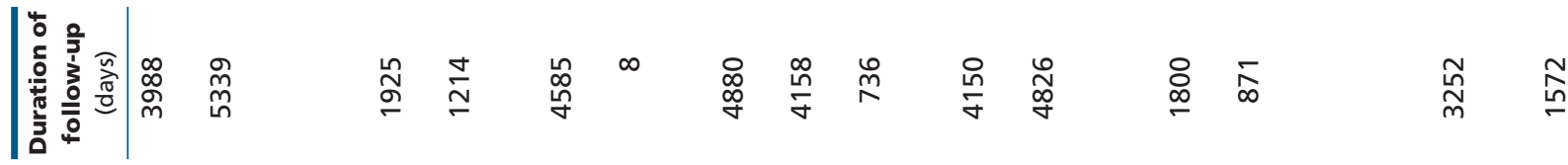
III

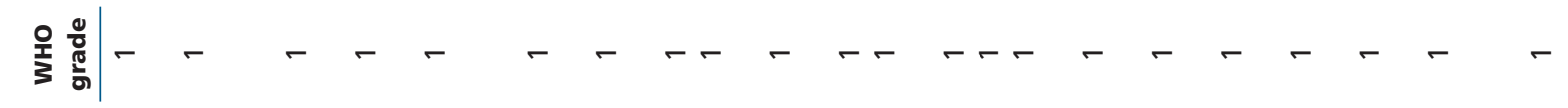

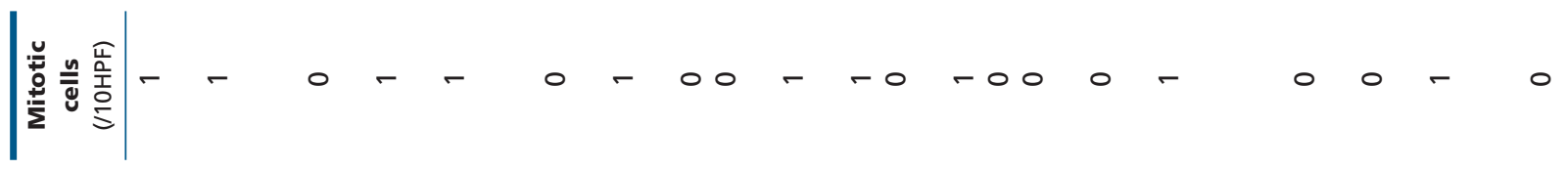

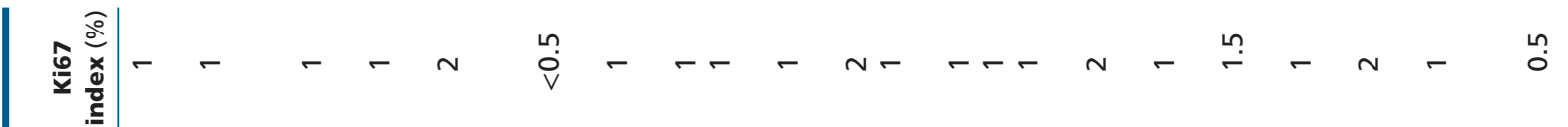

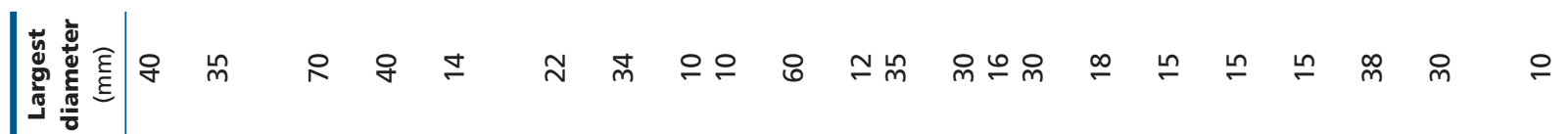

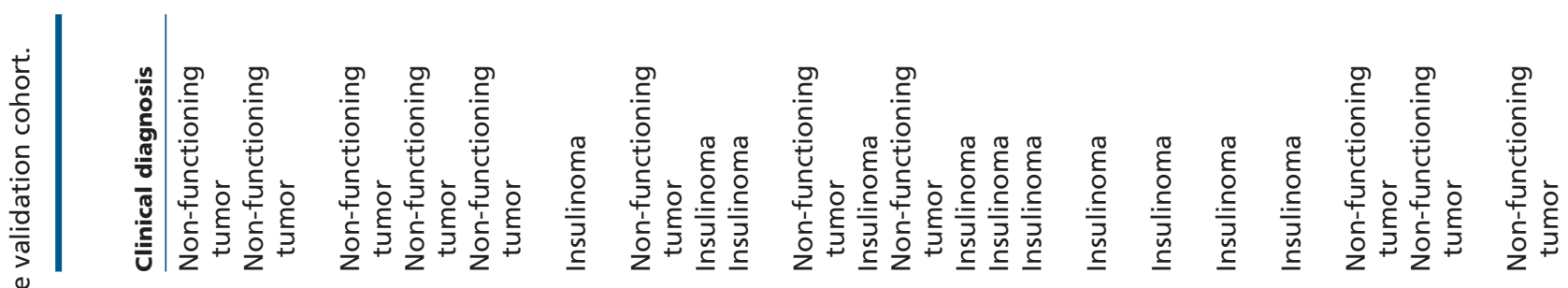

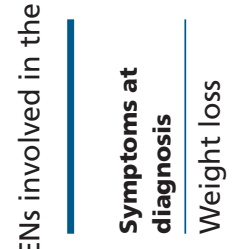

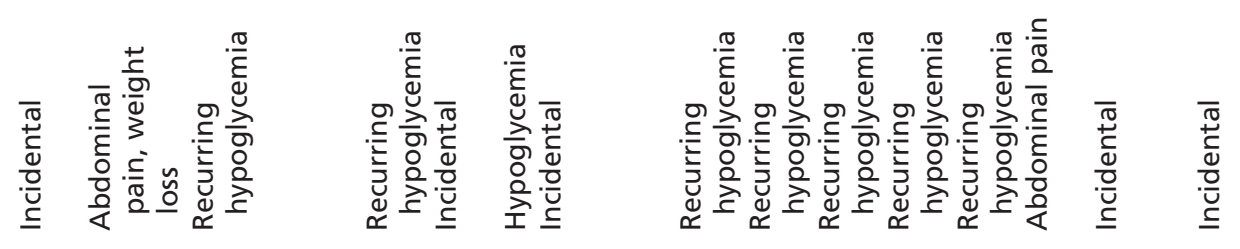

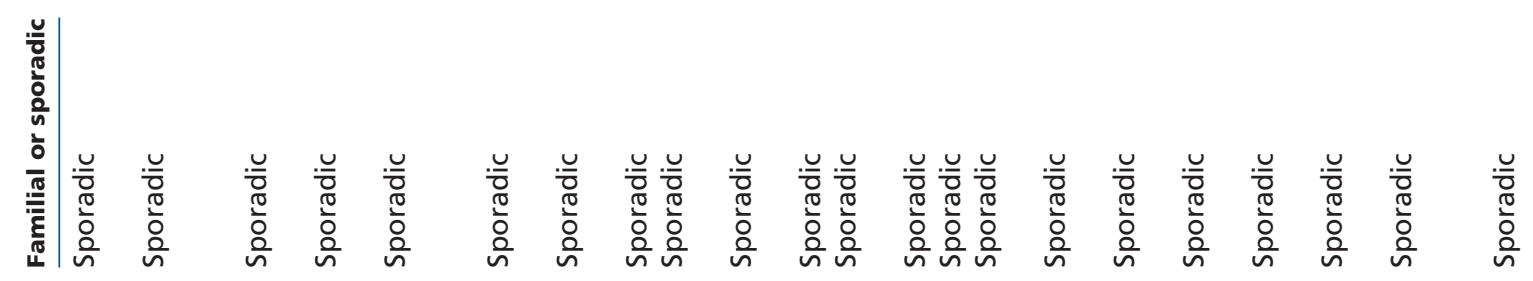

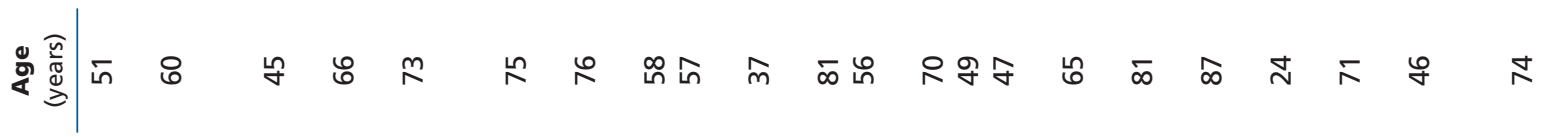
产 


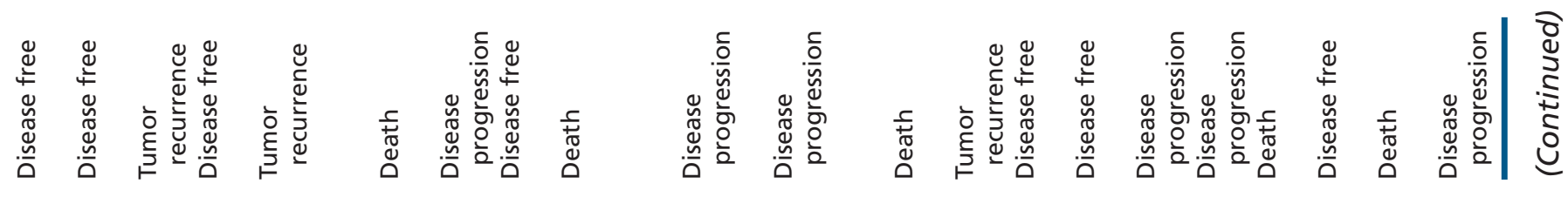

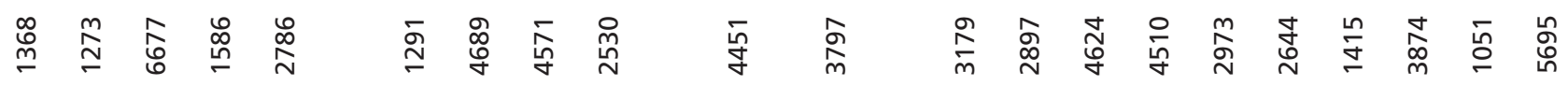

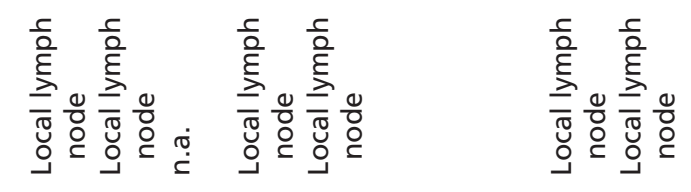

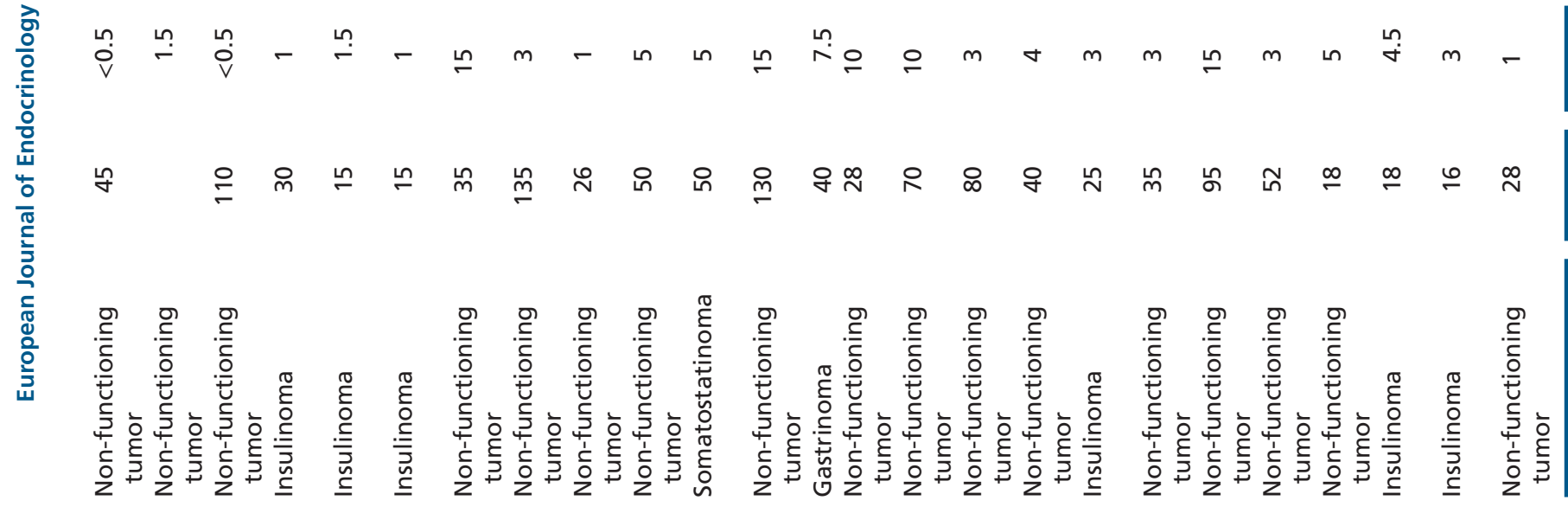

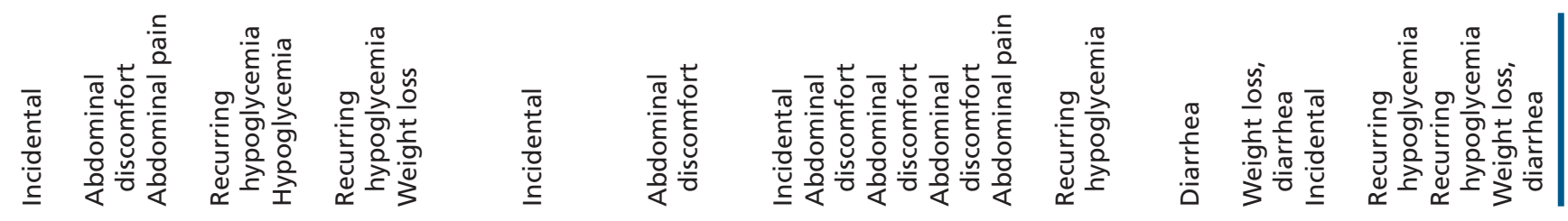

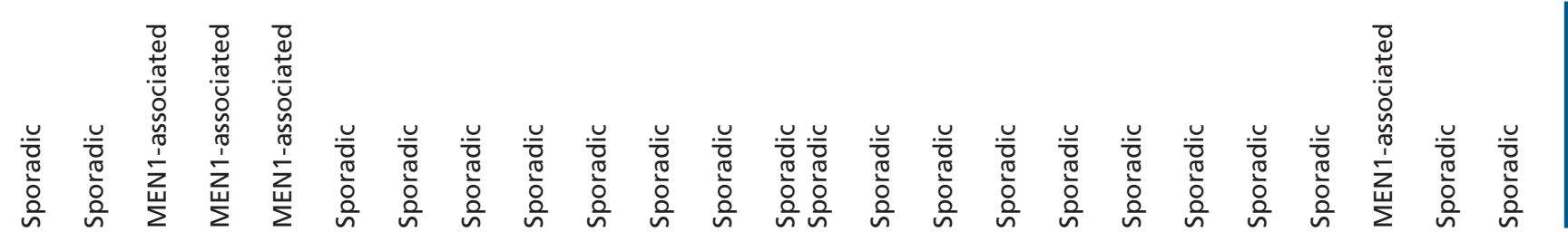

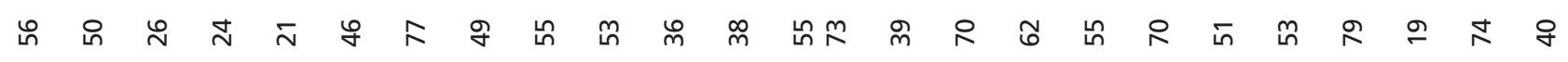

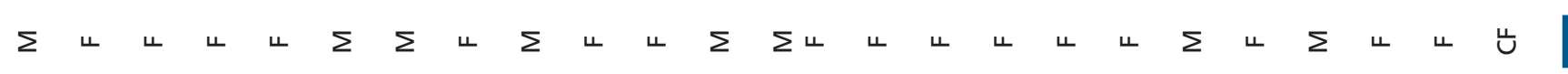

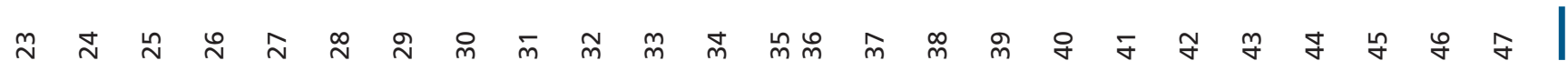




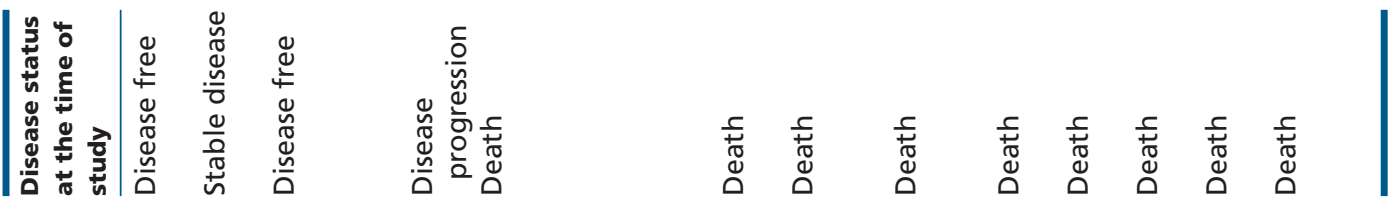

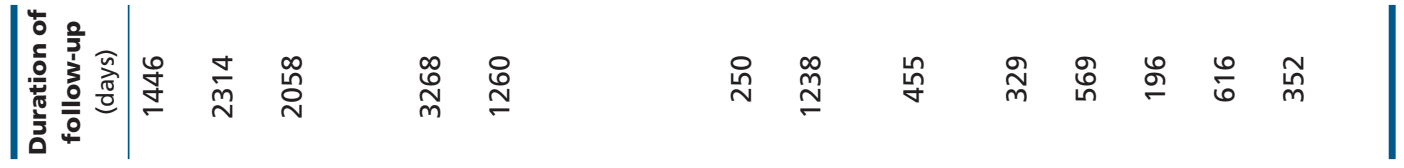

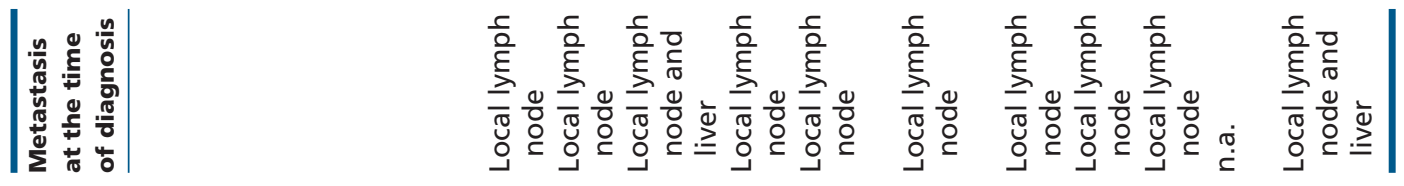

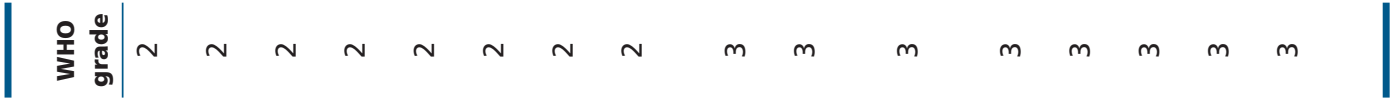

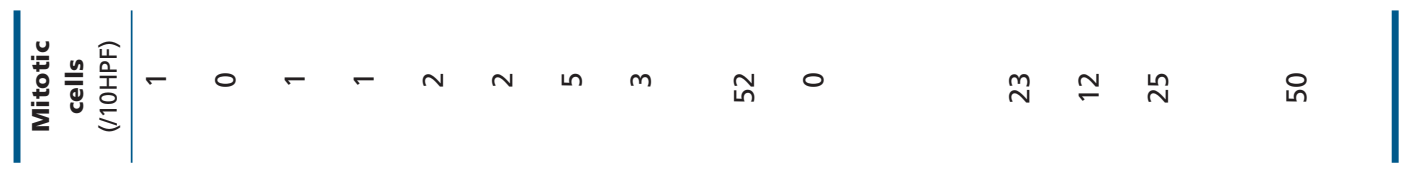

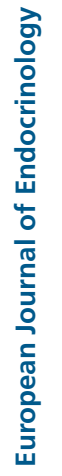

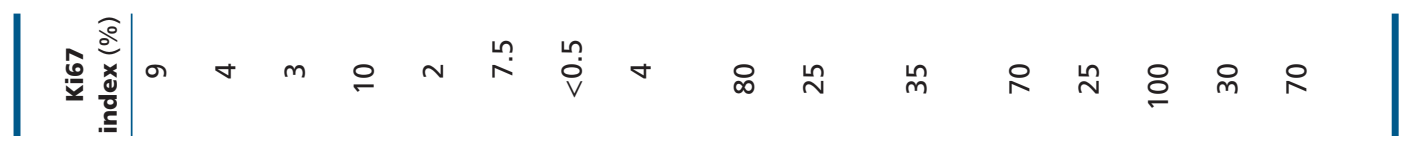

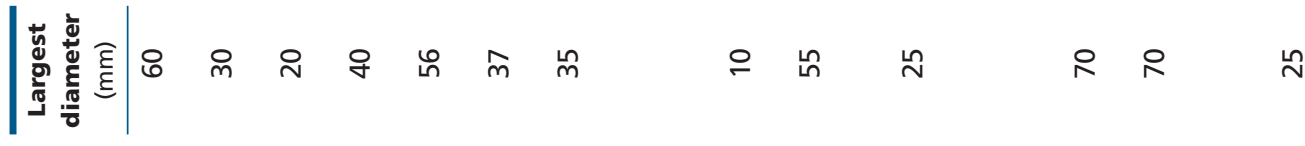

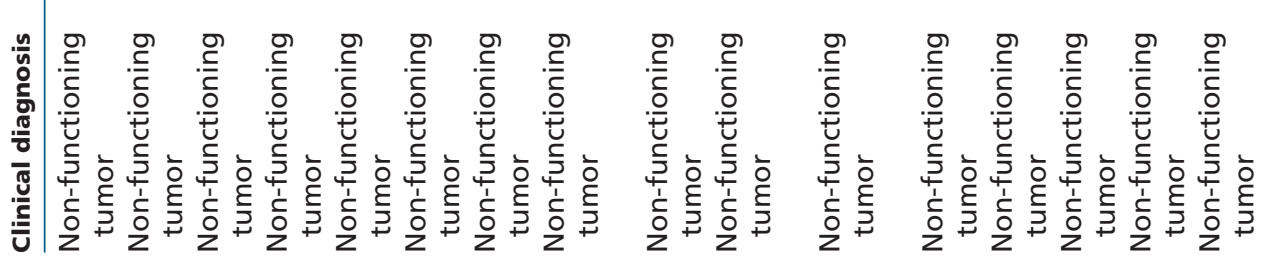

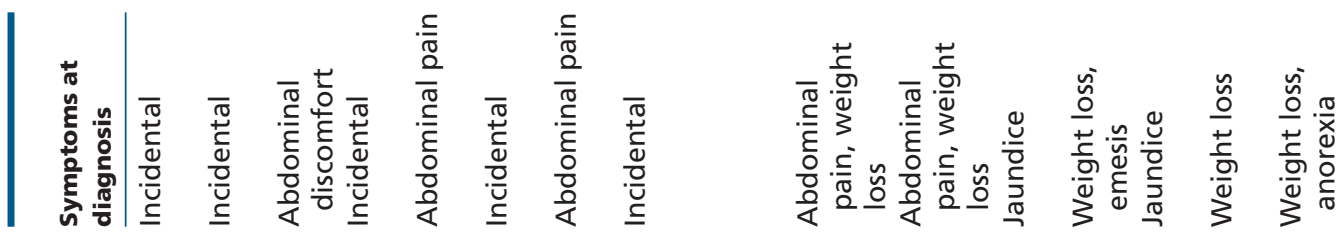

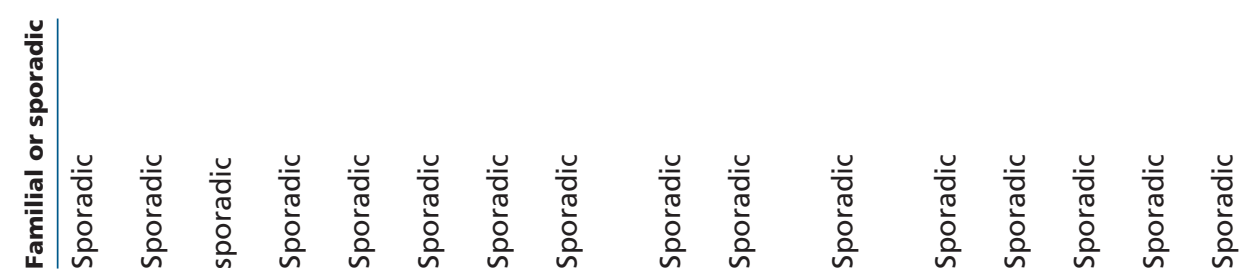

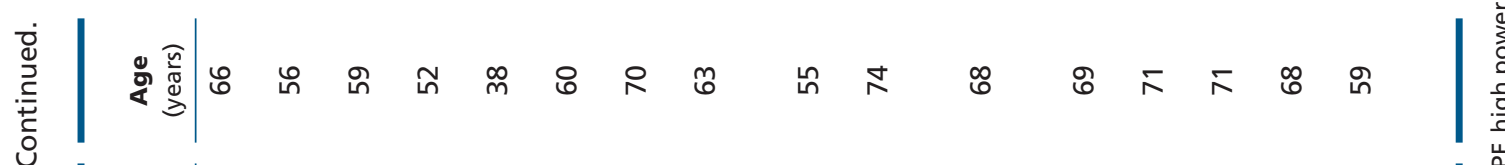

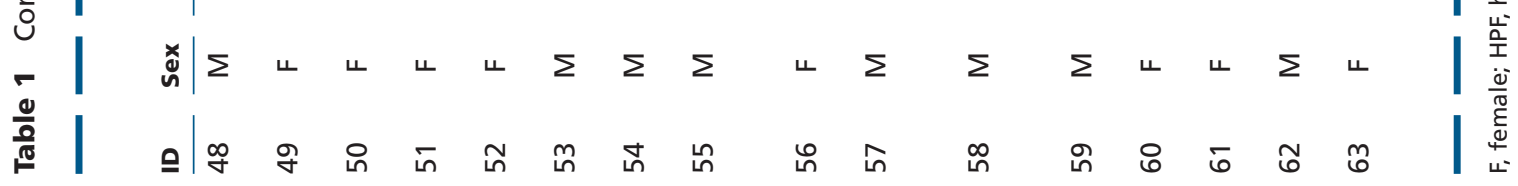




\section{Expression analysis of hsa-miR-21, hsa-miR-10a and hsa-miR-106b between non-metastatic and metastatic disease}

In our validation cohort, four patients had local lymph node and also liver, 14 patients had only local lymph node metastasis (totally 18 patients had metastatic disease) while 41 patients did not have metastasis at the time of diagnosis. We compared the expression levels of hsa-miR-21, hsa-miR-10a and hsa-miR-106b between patients with non-metastatic vs metastatic disease. Patients with metastatic disease had higher expression of hsa-miR-21 in the primary tumor $(-\Delta \mathrm{Ct}$ (non-metastatic): $3.22 \pm 1.27$ vs $-\Delta \mathrm{Ct}$ (metastatic): $4.08 \pm 1.64 P=0.0346$, note that the higher $-\Delta \mathrm{Ct}$ values correspond to higher expression), while there were no differences in the expression levels of hsa-miR-10a ( $-\Delta \mathrm{Ct}$ (non-metastatic): $-0.35 \pm 1.57$ vs $-\Delta \mathrm{Ct}$ (metastatic): $0.47 \pm 2.30 P=0.1229$ ) and hsa-miR-106b ( $-\Delta \mathrm{Ct}$ (non-metastatic): $1.21 \pm 1.31$ vs $-\Delta \mathrm{Ct}$ (metastatic): $1.80 \pm 1.70 P=0.1630)$ in this regard.

\section{Expression levels of hsa-miR-21, hsa-miR-10a and hsa-miR-106b have prognostic relevance in patients with PanNEN}

Follow-up of 49 patients has been conducted for $7.01 \pm 4.7$ years. Univariate analysis revealed that hsamiR-21, hsa-miR-10a and hsa-miR-106b are negative predictors of progression-free and overall survival; however, multivariate analysis confirmed only
hsa-miR-21 as an independent prognostic factor in PanNEN (Table 2, Panels A and B). Out of 49 patients during follow-up, 33 patients had disease progression (disease progression, tumor recurrence or death). In patients with disease progression, the expression levels of hsa-miR-10a and hsa-miR-106b were found to be negative predictors on the time to disease progression; however, no miRNAs were confirmed as independent prognostic factor (Table 2, Panel C).

\section{Discussion}

Due to their chemical stability, tissue specificity and pathogenic relevance, miRNAs are widely used biomarkers in health and disease. Minimally invasive sampling techniques facilitate early diagnosis and disease recurrence, which is of utmost importance regarding various diseases including cancer $(19,20)$. In particular, liquid biopsy from blood, saliva and urine may differentiate patients with different prognosis and contributes to histopathological diagnosis, as well $(19,20)$.

Although miRNA biomarkers are intensively sought in various types of cancer, only a few studies were directed to enlighten miRNA expression differences in PanNENs $(13,14,16)$, and prognostic relevance of only hsa-miR196a has been clearly proposed (16). Therefore, we aimed to reanalyze the results of the previously published and publicly available dataset of Roldo et al. (13) and validate our findings on the largest cohort in miRNA expression

Table 2 Survival analyses in the validation cohort. Follow-up information was available from 49 patients in the validation cohort. Cox proportional hazards survival regression models were fit for both univariate and multivariate analysis to determine the miRNA's effects on progression-free (Panel $A, n=49$ ) and overall survival (Panel $B, n=49$ ). Moreover, on patients with disease progression during follow-up additional Cox proportional hazards survival regression models were fit for both univariate and multivariate analysis to determine the miRNA's effects on time to disease progression (Panel C, $n=33$ ).

\begin{tabular}{|c|c|c|c|c|}
\hline \multirow[b]{2}{*}{ Factor } & \multicolumn{2}{|c|}{ Univariate analysis } & \multicolumn{2}{|c|}{ Multivariate analysis } \\
\hline & $\mathrm{HR}(95 \% \mathrm{Cl})$ & $P$-Value & $\mathrm{HR}(95 \% \mathrm{Cl})$ & $P$-Value \\
\hline \multicolumn{5}{|l|}{ A } \\
\hline hsa-miR-21 & $1.04(1.02-1.06)$ & 0.0002 & $1.03(1.01-1.05)$ & 0.0120 \\
\hline hsa-miR-10a & $1.10(1.04-1.16)$ & 0.0006 & $1.11(0.89-1.38)$ & 0.3556 \\
\hline hsa-miR-106b & 1.04 (1.02-1.07) & 0.0022 & $0.97(0.87-1.07)$ & 0.5249 \\
\hline \multicolumn{5}{|l|}{ B } \\
\hline hsa-miR-21 & $1.04(1.02-1.05)$ & 0.0001 & $1.03(1.00-1.05)$ & 0.0313 \\
\hline hsa-miR-10a & $1.16(1.05-1.28)$ & 0.0023 & $1.03(0.80-1.34)$ & 0.7997 \\
\hline hsa-miR-106b & 1.07 (1.03-1.12) & 0.0012 & $1.03(0.91-1.16)$ & 0.6428 \\
\hline \multicolumn{5}{|l|}{$\mathrm{C}$} \\
\hline hsa-miR-21 & 1.01 (1.00-1.03) & 0.1665 & $1.00(0.98-1.02)$ & 0.8792 \\
\hline hsa-miR-10a & 1.08 (1.02-1.14) & 0.0049 & $1.10(0.82-1.46)$ & 0.5246 \\
\hline hsa-miR-106b & 1.04 (1.01-1.07) & 0.0070 & $0.99(0.86-1.14)$ & 0.8981 \\
\hline
\end{tabular}

Bold letters indicate statistically significant associations $(P<0.05)$.

$95 \% \mathrm{Cl}, 95 \%$ confidence interval; $\mathrm{HR}$, hazard ratio. 
studies of PanNENs to date, specifically addressing the important issue of prognostic relevance.

Our in silico reanalysis revealed 19 miRNAs with altered expression between different tumor grades. Among these, the differential expression of hsa-miR20a and hsa-miR-21 in PanNENs has been confirmed by an independent study, as well (14). The hierarchical clustering rendered the discovery cohort samples into two clusters upon the expression profile of these 19 miRNAs successfully creating the lesser proliferative $\mathrm{C} 1$ and the more proliferative C2 clusters. Upon the qRT-PCR results, three (hsa-miR-21, hsa-miR-10a and hsa-miR-106b) out of five chosen miRNAs has been successfully validated as overexpressed in more proliferative tumors in an independent larger cohort of PanNENs, confirming the biological relevance of our in silico analysis.

In the validation cohort, hsa-miR-21 had the largest fold increase between tumor grades with elevated expression in Grade 3 PanNENs. Since the previous two large sample miRNA expression studies also associated hsa-miR-21 expression with PanNEN formation and more aggressive biological behavior $(13,14)$, currently, this miRNA seems to be most tightly related to PanNEN tumorigenesis. Most recently, a large case-control study assessing tumor and plasma miRNA expression of 320 cases found in accordance, that hsa-miR-21 expression was higher in patients with metastatic small intestine neuroendocrine tumor than in healthy controls (21). Additionally, high hsa-miR-21 expression associated with shorter overall survival (21). Confirming previous studies $(13,21)$, we also showed that higher hsamiR-21 expression in the primary tumor correlated with metastatic disease.

The importance of hsa-miR-21 as an onco-miR has been widely confirmed in several types of cancer (22); moreover, its targets have also been characterized (22), among which the negative correlation of hsa-miR-21 and its target programmed cell death 4 gene (PDCD4) has already been proposed by Roldo et al. (13). Moreover, the tumor suppressor protein PDCD4 has been confirmed as a target of hsa-miR-21 in breast cancer cells (23) and other important pro-apoptotic factors (FasL and PTEN) have also been confirmed as direct targets of hsa-miR-21 (24). Therefore, the inhibition of this pro-apoptotic pathway might contribute to cell survival and proliferation. Hsa-miR-21 has been shown to downregulate tumor suppressor PTEN resulting in cell proliferation in cervical (25) and colorectal (26) cancers as well. Additionally, upon targeting the tumor suppressor VHL hsa-miR-21 has been shown to promote the metastatic phenotype in cervical cancer (27). Most recently, co-delivery of gemcitabine and anti-miR-21 oligonucleotide resulted in synergistic antitumor effect in pancreatic cancer cells, paving the path for miR-21-targeted therapies (28).

The proto-oncogenic consequences of elevated expression of hsa-miR-106b has been observed in gastric cancer (29) and malignant melanoma (30), too. Additionally, the important cyclin-dependent kinaseinhibitor p21 has been confirmed to be a direct target of hsa-miR-106b $(29,30)$, while the downregulation of hsa-miR-106b promotes G1 phase arrest (30). Hsa-miR10a has been found to be overexpressed in non-small-cell lung cancer (NSCLC) (31). Additionally, hsa-miR-10a has been shown to directly target the tumor suppressor gene PTEN, contributing to the promotion of a more aggressive biological phenotype observed in NSCLC (31).

The most important finding of our study is the prognostic relevance of hsa-miR-21, hsa-miR-106b and hsa-miR-10a expression in PanNENs, among which hsamiR-21 has been shown to be an independent prognostic factor in the multivariate analysis in the largest cohort of PanNEN miRNA studies. Moreover, interestingly, in patients with disease progression, the higher expression of hsa-miR-10a and hsa-miR-106b, but not of hsa-miR-21 predicted earlier disease progression; however, neither of them was confirmed as an independent prognostic factor in this regard. In the next step, a prospective analysis of these miRNAs from body fluids upon different types of liquid biopsy in PanNEN patients may contribute to a more precise characterization of PanNENs before surgery providing new tools for precision oncology.

In conclusion, we detected and successfully validated the altered expression of hsa-miR-21, hsa-miR-106b and hsa-miR-10a on the largest PanNEN cohort for miRNA expression studies to date. Additionally, these miRNAs, especially hsa-miR-21, have prognostic relevance regarding progression-free and overall survival, which prompt us for further studies regarding its utilization in the early diagnosis of PanNENs.

Declaration of interest

The authors declare that there is no conflict of interest that could be perceived as prejudicing the impartiality of this study.

\section{Funding}

The authors acknowledge the financial support of the Hungarian Academy of Sciences ('Lendulet' 2013 grant awarded to A P) and of the Hungarian Scientific Research Grant (OTKA, K125231 to A P). 
Author contribution statement

A P conceived the study. V K G and A P designed research. V K G performed in silico analysis. $\mathrm{K} B$ performed immunohistochemistry and histopathologically analyzed samples. V K G performed qRT-PCR measurements. V K G, A K, K B, P I and A P analyzed data. V K G drafted the manuscript. All authors acknowledged the final version of the manuscript.

\section{Acknowledgements}

The authors would like to express their gratitude to the recently deceased Prof. Károly Rácz, founder of the Endocrine Genetics Laboratory of the 2nd Department of Medicine, Semmelweis University, without whom the research presented here would not have been possible. The authors would like to acknowledge the excellent technical assistance of Anna Szentpéteri and Zsófia Vásárhelyi regarding RNA isolation and qRT-PCR measurements.

\section{References}

1 Halfdanarson TR, Rabe KG, Rubin J \& Petersen GM. Pancreatic neuroendocrine tumors (PNETs): incidence, prognosis and recent trend toward improved survival. Annals of Oncology 200819 1727-1733. (https://doi.org/10.1093/annonc/mdn351)

2 Yao JC, Hassan M, Phan A, Dagohoy C, Leary C, Mares JE, Abdalla EK, Fleming JB, Vauthey JN, Rashid A et al. One hundred years after 'Carcinoid': epidemiology of and prognostic factors for neuroendocrine tumors in 35825 cases in the United States. Journal of Clinical Oncology 200826 3063-3072. (https://doi.org/10.1200/ JCO.2007.15.4377)

3 Jensen RI, Berna MJ, Bingham DB \& Norton JA. Inherited pancreatic endocrine tumor syndromes: advances in molecular pathogenesis, diagnosis, management, and controversies. Cancer 2008113 1807-1843. (https://doi.org/10.1002/cncr.23648)

4 Klimstra DS, Modlin IR, Coppola D, Lloyd RV \& Suster S. The pathologic classification of neuroendocrine tumors a review of nomenclature, grading, and staging systems. Pancreas 201039 707-712. (https://doi.org/10.1097/MPA.0b013e3181ec124e)

5 Jiao YC, Shi CJ, Edil BH, de Wilde RF, Klimstra DS, Maitra A, Schulick RD, Tang LH, Wolfgang CL, Choti MA et al. DAXX/ATRX, MEN1, and mTOR pathway genes are frequently altered in pancreatic neuroendocrine tumors. Science 2011331 1199-1203. (https://doi. org/10.1126/science.1200609)

6 Missiaglia E, Dalai I, Barbi S, Beghelli S, Falconi M, della Peruta M, Piemonti L, Capurso G, Di Florio A, delle Fave G et al. Pancreatic endocrine tumors: expression profiling evidences a role for AKTmTOR pathway. Journal of Clinical Oncology 201028 245-255. (https://doi.org/10.1200/JCO.2008.21.5988)

7 Scarpa A, Chang DK, Nones K, Corbo V, Patch AM, Bailey P, Lawlor RT, Johns AL, Miller DK, Mafficini A et al. Whole-genome landscape of pancreatic neuroendocrine tumours. Nature $2017 \mathbf{5 4 3}$ 65-71. (https://doi.org/10.1038/nature21063)

8 Valencia-Sanchez MA, Liu J, Hannon GJ \& Parker R. Control of translation and mRNA degradation by miRNAs and siRNAs. Genes and Development 200620 515-524. (https://doi.org/10.1101/ gad.1399806)

9 Shenouda SK \& Alahari SK. MicroRNA function in cancer: oncogene or a tumor suppressor? Cancer and Metastasis Reviews 200928 369-378. (https://doi.org/10.1007/s10555-009-9188-5)

10 Grolmusz VK, Borka K, Kovesdi A, Nemeth K, Balogh K, Dekany C, Kiss A, Szentpeteri A, Sarman B, Somogyi A et al. MEN1 mutations and potentially MEN1-targeting miRNAs are responsible for menin deficiency in sporadic and MEN1 syndrome-associated primary hyperparathyroidism. Virchows Archiv 2017471 401-411. (https:// doi.org/10.1007/s00428-017-2158-3)
11 Tombol Z, Eder K, Kovacs A, Szabo PM, Kulka J, Liko I, Zalatnai A, Racz G, Toth M, Patocs A et al. MicroRNA expression profiling in benign (sporadic and hereditary) and recurring adrenal pheochromocytomas. Modern Pathology 201023 1583-1595. (https:// doi.org/10.1038/modpathol.2010.164)

12 Cheng WT, Rosario R, Muthukaruppan A, Wilson MK, Payne K, Fong PC, Shelling AN \& Blenkiron C. MicroRNA profiling of ovarian granulosa cell tumours reveals novel diagnostic and prognostic markers. Clinical Epigenetics 20179 72. (https://doi.org/10.1186/ s13148-017-0372-0)

13 Roldo C, Missiaglia E, Hagan JP, Falconi M, Capelli P, Bersani S, Calin GA, Volinia S, Liu CG, Scarpa A et al. MicroRNA expression abnormalities in pancreatic endocrine and acinar tumors are associated with distinctive pathologic features and clinical behavior. Journal of Clinical Oncology 200624 4677-4684. (https://doi. org/10.1200/JCO.2005.05.5194)

14 Thorns C, Schurmann C, Gebauer N, Wallaschofski H, Kumpers C, Bernard V, Feller AC, Keck T, Habermann JK, Begum N et al. Global microRNA profiling of pancreatic neuroendocrine neoplasias. Anticancer Research 201434 2249-2254.

15 Sadanandam A, Wullschleger S, Lyssiotis CA, Grotzinger C, Barbi S, Bersani S, Korner J, Wafy I, Cini AM, Lawlor RT et al. A cross-species analysis in pancreatic neuroendocrine tumors reveals molecular subtypes with distinctive clinical, metastatic, developmental, and metabolic characteristics. Cancer Discovery 20155 1296-1313. (https://doi.org/10.1158/2159-8290.CD-15-0068)

16 Lee YS, Kim H, Kim HW, Lee JC, Paik KH, Kang J, Kim J, Yoon YS, Han HS, Sohn I et al. High expression of microRNA-196a indicates poor prognosis in resected pancreatic neuroendocrine tumor. Medicine 201594 e2224. (https://doi.org/10.1097/ MD.0000000000002224)

17 WHO Classification of Tumours of Endocrine Organs, WHO/IARC Classification of Tumours. WHO Press, 2017.

18 Grolmusz VK, Toth EA, Baghy K, Liko I, Darvasi O, Kovalszky I, Matko J, Racz K \& Patocs A. Fluorescence activated cell sorting followed by small RNA sequencing reveals stable microRNA expression during cell cycle progression. BMC Genomics 201617412. (https://doi.org/10.1186/s12864-016-2747-6)

19 Chabre O, Libe R, Assie G, Barreau O, Bertherat J, Bertagna X, Feige JJ $\&$ Cherradi N. Serum miR-483-5p and miR-195 are predictive of recurrence risk in adrenocortical cancer patients. Endocrine-Related Cancer 201320 579-594.

20 Di Meo A, Bartlett J, Cheng YF, Pasic MD \& Yousef GM. Liquid biopsy: a step forward towards precision medicine in urologic malignancies. Molecular Cancer 201716 80. (https://doi.org/10.1186/ s12943-017-0644-5)

21 Bowden M, Zhou CW, Zhang S, Brais L, Rossi A, Naudin L, Thiagalingam A, Sicinska E \& Kulke MH. Profiling of metastatic small intestine neuroendocrine tumors reveals characteristic miRNAs detectable in plasma. Oncotarget $2017854331-54344$.

22 Pfeffer SR, Yang CH \& Pfeffer LM. The role of miR-21 in cancer. Drug Development Research 201576 270-277. (https://doi.org/10.1002/ ddr.21257)

23 Frankel LB, Christoffersen NR, Jacobsen A, Lindow M, Krogh A \& Lund AH. Programmed cell death 4 (PDCD4) is an important functional target of the microRNA miR-21 in breast cancer cells. Journal of Biological Chemistry 2008283 1026-1033. (https://doi. org/10.1074/jbc.M707224200)

24 Hu JZ, Huang JH, Zeng L, Wang G, Cao M \& Lu HB. Anti-apoptotic effect of microRNA-21 after contusion spinal cord injury in rats. Journal of Neurotrauma 201330 1349-1360. (https://doi.org/10.1089/ neu.2012.2748)

25 Peralta-Zaragoza O, Deas J, Meneses-Acosta A, De la O-Gómez F, Fernandez-Tilapa G, Gomez-Ceron C, Benitez-Boijseauneau O, Burguete-Garcia A, Torres-Poveda K, Bermudez-Morales VH et al. Relevance of miR-21 in regulation of tumor suppressor gene PTEN in 
human cervical cancer cells. BMC Cancer 201616 215. (https://doi. org/10.1186/s12885-016-2231-3)

26 Wu Y, Song Y, Xiong Y, Wang X, Xu K, Han B, Bai Y, Li L, Zhang Y $\&$ Zhou L. MicroRNA-21 (Mir-21) promotes cell growth and invasion by repressing tumor suppressor PTEN in colorectal cancer. Cellular Physiology and Biochemistry 201743 945-958. (https://doi. org/10.1159/000481648)

27 Cai L, Wang W, Li X, Dong T, Zhang Q, Zhu B, Zhao H \& Wu S. MicroRNA-21-5p induces the metastatic phenotype of human cervical carcinoma cells in vitro by targeting the von Hippel-Lindau tumor suppressor. Oncology Letters 201815 5213-5219.

28 Li Y, Chen Y, Li J, Zhang Z, Huang C, Lian G, Yang K, Chen S, Lin Y, Wang $L$ et al. Co-delivery of microRNA-21 antisense oligonucleotides and gemcitabine using nanomedicine for pancreatic cancer therapy.
Cancer Science 2017108 1493-1503. (https://doi.org/10.1111/ cas.13267)

29 Kim YK, Yu J, Han TS, Park SY, Namkoong B, Kim DH, Hur K, Yoo MW, Lee HJ, Yang HK et al. Functional links between clustered microRNAs: suppression of cell-cycle inhibitors by microRNA clusters in gastric cancer. Nucleic Acids Research 200937 1672-1681. (https:// doi.org/10.1093/nar/gkp002)

30 Prasad R \& Katiyar SK. Down-regulation of miRNA-106b inhibits growth of melanoma cells by promoting G1-phase cell cycle arrest and reactivation of p21/WAF1/Cip1 protein. Oncotarget 20145 10636-10649.

31 Yu T, Liu L, Li J, Yan M, Lin H, Liu Y, Chu D, Tu H, Gu A \& Yao M. MiRNA-10a is upregulated in NSCLC and may promote cancer by targeting PTEN. Oncotarget 20156 30239-30250.

Received 10 April 2018

Revised version received 27 June 2018

Accepted 12 July 2018 